\title{
The ticking time bomb: Using eye-tracking methodology to capture attentional processing during gradual time constraints
}

\author{
Ana M. Franco-Watkins ${ }^{1}$ • Matthew E. Davis ${ }^{1}$ - Joseph G. Johnson ${ }^{2}$
}

Published online: 9 August 2016

(C) The Psychonomic Society, Inc. 2016

\begin{abstract}
Many decisions are made under suboptimal circumstances, such as time constraints. We examined how different experiences of time constraints affected decision strategies on a probabilistic inference task and whether individual differences in working memory accounted for complex strategy use across different levels of time. To examine information search and attentional processing, we used an interactive eye-tracking paradigm where task information was occluded and only revealed by an eye fixation to a given cell. Our results indicate that although participants change search strategies during the most restricted times, the occurrence of the shift in strategies depends both on how the constraints are applied as well as individual differences in working memory. This suggests that, in situations that require making decisions under time constraints, one can influence performance by being sensitive to working memory and, potentially, by acclimating people to the task time gradually.
\end{abstract}

Keywords Decision making $\cdot$ Strategy $\cdot$ Working memory · Attention · Eye tracking

Time constraints can impact both simple (e.g., forgoing breakfast after waking up late) and complex (e.g., finding a new place to live in a week instead of 2 months) decisions. Imposing a time constraint can affect which strategy is

The research was supported in part by grant SES-0851990 from the National Science Foundation.

Ana M. Franco-Watkins

afrancowatkins@auburn.edu

1 Department of Psychology, Auburn University, Auburn, AL, USA

2 Department of Psychology, Miami University, Oxford, OH, USA implemented such that a person might engage in a simpler strategy and accept an apartment that seems "good enough" when less time is available, rather than engaging in a more complex strategy to find the "best" alternative. In general, reducing the time allotted to make decisions has been found to impact both choice processes and outcomes (Svenson \& Maule, 1993). Under time constraints, people typically acquire less information or attempt to acquire a comparable amount of information but at a quicker rate (Ben-Zur \& Breznitz, 1981; Johnson, Zhou, Koop, \& Franco-Watkins, manuscript submitted for publication; Kerstholt, 1995; Payne, Bettman, \& Johnson, 1988). Additionally, people often employ selective information searches by focusing on the more important information (Böckenholt \& Kroeger, 1993; Johnson, Zhou, Koop, \& Franco-Watkins, manuscript submitted for publication; Kerstholt, 1995; Payne et al., 1988; Wright, 1974) and are more likely to acquire information within attributes to gain at least some information about each option (Maule, 1994; Maule, Hockey, \& Bdzola, 2000; Payne et al., 1988). Time constraints are integral to understanding decision-making strategies and how different strategies guide information search and, ultimately, one's choices. The purpose of the current paper was to account for how people attend to and process information during decision making under time pressure.

To date, most time constraint research has involved a relatively coarse time induction where time is empirically applied at two different levels (i.e., high/low or present/absent) (Rieskamp \& Hoffrage, 2008; Weenig \& Maarleveld, 2002), although a few studies have introduced three levels of time (Glöckner \& Betsch, 2008), allowing only marginally better localization of performance changes (for a notable exception, see Johnson, Zhou, Koop, \& Franco-Watkins, manuscript submitted for publication). However, time constraints in many real-world settings tends to lessen or intensify in an 
incremental manner rather than an all or none manner. For example, time intensifies incrementally as a deadline approaches or can lessen if the deadline is moved to a later date. Accordingly, it is important to understand whether the application of the stressor (lessening or intensifying the time to choose) affects how people attend to and process information and the impact these stressors have on decision strategies.

We sought to build and extend upon decision-making research by using a graded time manipulation (i.e., $3 \mathrm{~s}, 6 \mathrm{~s}, 9 \mathrm{~s}$, $12 \mathrm{s.} 15 \mathrm{~s}$, and $18 \mathrm{~s})^{1}$ within-participants and examined whether the application of the stressor either by increasing or decreasing time to choose (between-participants) affected strategy use and subsequent choice. Specifically, we focused on two particular strategies well represented in decision research. First, we considered a simple lexicographic (LEX) strategy that involves searching across attributes (cues) in order of their importance (validity) and selecting an option based on the first attribute to discriminate between choice options (i.e., alternatives). Second, we examined a more complex strategy, such as a weighted additive (WADD) that requires some calculation, namely, for each alternative, a weighted sum of attribute values is computed and then the decision maker selects the option with the highest sum. Past research has demonstrated that people are able to integrate cue information and use WADD strategies during the presence of lenient or moderate time constraints (Glöckner \& Betsch, 2008). To our knowledge, previous research has not examined whether the application of a stressor (i.e., increasing or decreasing time) differentially affects the decision-making process. Furthermore, previous research did not examine the use of multiple levels of time to determine the juncture where decision-making strategies may shift.

Although the presence time constraints can globally affect how one attends to and processes information, we propose that working memory might mediate information processing, especially in the presence of a stressor. Working memory allows one to process current information (overt attention) while maintaining relevant information in mind (covert attention) and ignoring or discarding irrelevant (e.g., stressor-induced) or distracting information. Furthermore, working memory operates as a limited-capacity mechanism of attentional control and manages higher-level cognitive functions, such as decision making (Baddeley \& Hitch, 1974; Baddeley, 2002). Individual differences in working memory often are viewed as the ability to control attention, including maintaining goalrelevant information and inhibiting attention on irrelevant information (Engle, 2002). Generally, there is a performance advantage for those with higher working memory (WM) compared with those with lower WM across a variety of cognitive

\footnotetext{
${ }^{1}$ Our previous research titrating time pressure at multiple levels suggests that these levels would be most diagnostic. Specifically, Johnson et al. (2015) used 6-second intervals from 36 to six seconds, and found most of the effects occurred below 18 seconds.
}

tasks, including perceptual (Kane, Bleckley, Conway, \& Engle, 2001; Awh, Vogel, \& Oh, 2006), goal-driven encoding and processing (Sanchez \& Wiley, 2006), memory retrieval (Kane \& Engle, 2000; Unsworth, Brewer, \& Spillers, 2013), and hypothesis generation (Dougherty \& Hunter, 2003; Dougherty \& Sprenger, 2006, Sprenger et al., 2011) tasks.

Individuals higher in WM can usually engage in more complex and/or rule-based processing compared with those with lower WM (Beach \& Mitchell, 1978; Beilock \& DeCaro, 2007). WM also facilitates category learning (Lewandowsky, 2011) as well as information acquisition and strategy in simple decision tasks (Rakow, Newell, \& Zougkou, 2010). Individual differences in working memory have emerged during the presence of a stressor, such as high performance pressure (i.e., being videotaped and performance accountability) or a dualtask procedure (i.e., completing a secondary task alongside of the primary task). The addition of a stressor can minimize and/ or equate the differences between higher and lower WM individuals that are typically found when no stressor is present (Beilock \& Carr, 2005; Kane \& Engle, 2000). Beilock and colleagues (Beilock \& Carr, 2005; Beilock \& DeCaro, 2007) found that higher WM individuals applied simpler strategies during a high performance pressure situation, where math/ problem-solving performance decreased to a level comparable to lower WM individuals. The performance decrement for higher WM individuals in the presence of a stressor is presumed to be based on attentional resources: when no stressor is present these individuals have an advantage in terms of attentional processing, however, in the presence of a stressor, higher WM individuals allocate attentional resources to the actual stressor, whereas lower WM individuals tend to allocate their limited attentional resources to the task (Beilock \& Carr, 2005). Accordingly, we examined whether or not individual differences in working memory accounted for attentional processing and strategy use variations across the different levels of time constraints. Furthermore, we examined if the application of the stressor (increasing or lessening time) impacted processing and strategy usage, and whether interactions with working memory were present.

\section{Eye tracking}

Decision research has utilized eye-tracking methods to better understand the mechanism underlying the decision processes in probabilistic inference tasks (Franco-Watkins \& Johnson, 2011a; Glöckner \& Betsch, 2008; Lohse \& Johnson, 1996), risky choice (Fiedler \& Glöckner, 2012; Franco-Watkins \& Johnson, 2011b), intertemporal choice (Franco-Watkins, Mattson \& Jackson, 2016), as well as consumer behavior (Russo \& Leclerc, 1994; Russo \& Rosen, 1975). Because we are interested in deliberate attentional processing and decision making, we used an 
interactive eye-tracking methodology, the decision moving window (Franco-Watkins \& Johnson, 2011a; 2011b). In this methodology, information is occluded from view until a person fixates on a specific piece of information (i.e., cell). This requires participants to retain information from previous cells, while acquiring additional information, thereby requiring working memory resources during decision making. As such, this method allowed us to determine whether overt attentional processing measures captured with eye tracking (e.g., fixations, duration, cells opened, etc.) can explain processing and strategy use during time pressure and whether individual differences in working memory factored into strategy use and choices.

Generally, conventional wisdom suggests that imposing severe time restrictions reduces one's ability to examine or use information in a complex decision, thereby affecting one's ability to engage in complex strategies (Payne et al., 1988; Rieskamp \& Hoffrage, 1999). Thus, we anticipated a switch from complex to simpler strategies during the stricter time constraints given that our method (interactive eye tracking) required deliberate attentional processing. The use of a LEX strategy uses fewer attentional resources because an individual can acquire fewer pieces of information, have fewer fixations, and rely more on an attributewise search. Conversely, the use of a WADD strategy necessitates using all information, thereby acquiring more cells, having more fixations, and relying more on an alternative-wise search to support calculations of all attributes for a given alternative. Furthermore, if working memory factors into the ability to use and maintain complex strategies, then higher WM individuals should be more prone to use a WADD strategy by virtue of a greater ability to control attentional resources (Beach \& Mitchell, 1978), especially when time restrictions are lenient or time is not restricted. Because working memory influences how individuals are able to control attention and acquire visual information, higher WM individuals are able to acquire visual information more quickly when distractions are present (Kane et al., 2001). Recent work by Ashby and Rakow (2014) demonstrated that individuals with greater cognitive capacity sought and utilized more information in a decision sampling paradigm. As such, the increased ability to acquire visual information may benefit those with higher WM and allow them to obtain more information within the time constraints, facilitating use of a WADD strategy. Perhaps due to poorer attentional control, lower WM individuals may be unable to acquire the same amount of information and thereby may be more apt to use a simpler LEX strategy.

However, the application of the stressor in a gradual manner might differentially affect strategy use. When the stressor gradually increases, higher WM might have the ability to adapt to these increasing demands and continue using their initial strategies even when time becomes more restrictive. Alternatively, they might be affected by the stressor to the same degree as lower WM individuals, perhaps due to the task demands co-opting the resource advantage of higher WM individuals (similar to the performance pressure research). In contrast, gradually lessening the stressor may result in simpler strategies for all individuals initially because of the strict time constraints experience initially, where not even the higher WM individuals are able to apply complex strategies. Nevertheless, individual differences in working memory can affect time estimation such that higher WM individuals are better at estimating time than lower WM (Fink \& Neubauer, 2005). In the current study, if higher WM individuals are better able to estimate their time available, they may quickly adapt to the time constraints and perhaps shift to using a WADD strategy sooner. Thus, when more time is available, higher WM individuals might be better able to apply available attentional resources and use a complex strategy sooner than lower WM individuals, who may continue to use their initial, simpler strategy.

\section{Method}

\section{Participants}

A total of 129 undergraduate students with normal or corrected vision completed the experiment in exchange for extra credit towards a psychology course. Participants were incentivized as they were told $\$ 50$ would be given to the highest two performers; however, students were not instructed as to which strategy would yield the highest performance.

\section{Design}

The experiment used a mixed factorial design with 6 levels of decision time $(3 \mathrm{~s}, 6 \mathrm{~s}, 9 \mathrm{~s}, 12 \mathrm{~s}, 15 \mathrm{~s}$, and $18 \mathrm{~s})$ as a withinparticipants variable. Participants completed the task under incrementally decreasing time (18 s, $15 \mathrm{~s}, 12 \mathrm{~s}, 9 \mathrm{~s}, 6 \mathrm{~s}, 3 \mathrm{~s})$ or increasing time $(3 \mathrm{~s}, 6 \mathrm{~s}, 9 \mathrm{~s}, 12 \mathrm{~s}, 15 \mathrm{~s}, 18 \mathrm{~s})$ blocks as a between-participants factor. Additionally, all participants completed a baseline block with no time pressure (no TP) directly before or after completing the most lenient time phase (18 s), depending on their assigned order.

We used the operation span task as a measure of working memory. Research examining working memory and stress (e.g., performance pressure) typically classifies higher and lower groups based on a median split of working memory scores (Beilcok \& Carr, 2005). The current paper classified higher WM $(M=58.45, S D=7.78)$ or lower WM participants $(M=32.02, S D=10.98)$ using the median working memory score of 44 (range $0-75$ ). Using tertiles to split working memory groups resulted in 
similar results, and we used the median ${ }^{2}$ split to include all participants. The increasing time condition encompassed 31 higher WM and 33 lower WM participants and the decreasing time condition consisted of 33 higher WM and 32 lower WM. Working memory scores were similar between increasing and decreasing time conditions, $t(127)=-0.76$, $p>0.05$.

\section{Apparatus}

We used the Tobii 1750 eye tracker (17" monitor, screen: $1024 \times 768$ pixels) with E-prime (Psychology Software Tools). Participants were calibrated to device using Tobii Clearview software (Tobii Technology, Danderyd, Sweden).

\section{Materials}

Decision task The decision task consisted of a probabilistic inference task used in prior work (Franco-Watkins \& Johnson, 2011a); there were a series of $3 \times 4$ matrix tables where participants made a probabilistic inferential decision about which of three movies (A, B, or C) grossed the highest box office revenue. Four attributes: star power, big production budget, PG-13 rating, and original screenplay, with each attribute corresponding to specific predictive validities: $0.80,0.70,0.60$, and 0.50 , respectively were indicated in the task. ${ }^{3}$ Within each matrix table, row headings represented movie alternatives (e.g., Movie A) and column headings represented attributes (e.g., Budget). Individual cells corresponded to specific attribute binary values denoting the presence $(+)$ or absence $(-)$ of an attribute for a given alternative.

Attributes appeared in a fixed left-to-right order by decreasing predictive validity. Each table cell represented a unique area of interest (AOI). All information cells, as well as alternative and attribute labels, were identical in size (18\% width and $16 \%$ height of the screen as presented in E-prime or $184 \times$ 123 pixels). Each cell represented a unique area of interest (AOI). The binary cues used as stimuli were images constructed to fit the AOI window size (see Franco-Watkins \& Johnson, 2011a for further details). All stimuli were prominently displayed to encompass most of the AOI.

Participants used interactive eye-tracking, the decision moving window, to search for cues within cells (Franco-

\footnotetext{
${ }^{2}$ Working memory measures represent a range of scores; however, it is not usually denoted as an interval or ratio score given that differences between two scores are not as meaningful and usually treated as a categorical variable to indicate general working memory capacity. Given the complexity of our design with between and within-subjects variables and the dependent variable consisting of only four responses per cell, we did not have enough power to use working memory as a continuous measure in our statistical analyses.

${ }^{3}$ These attributes reflect real-world ordinal relationship of predictive earnings; however, the validities used in the task were changed to construct theoretically diagnostic stimuli.
}

Watkins \& Johnson, 2011a; 2011b). Labels for each option and attribute remained visible on the screen; however, cell information was hidden until the subject's eye fixated on a target cell (henceforth known as a fixation).

Figure 1a presents a schematic of a decision task trial. The allotted time to complete the task was blocked, with each block including five matrices (four experimental trials presented in Fig. $1 b$ and one filler trial not included). Matrices were presented in different configurations per block ${ }^{4}$ and presented in random order per each amount of time resulting in a total of 35 trials (including the baseline block). The stimuli for the experiments were created by first designing tables diagnostic between WADD and LEX decision strategies (Fig. 1b) such that each movie choice represented only one of the three possible strategies: WADD, LEX, or neither strategy. Using a WADD strategy in this task involves taking a cumulative predictive validity for each movie and selecting the movie with the highest sum, whereas a LEX strategy is characterized by choosing a movie based on the first attribute to discriminate from the other movies.

Working memory We used the automated operation span task (O-span; Unsworth, Heitz, Schrock, \& Engle, 2005) to measure the working memory of each participant. The O-span consists of 75 sets of simple mathematical verifications (e.g., $8 / 4+3=7$ ?) coupled with a letter. Participants indicated whether or not the mathematical operation is true or false, then a letter is presented for later recall. They complete a succession of operation plus letter pairings (varying from 3 to 7 pairings in a series) before being prompted to recall the letters in the correct serial position. O-span scores represent the total number of correctly recalled letters in the correct serial position with larger scores indicative of higher working memory ability. As per guidelines for working memory tasks, participants who did not achieve $85 \%$ accuracy on the math problems were removed from analyses (Unsworth et al., 2005).

\section{Procedure}

Once participants completed the O-span task, they were placed in front of the eye-tracker apparatus and then calibrated to the eye-tracker device. Participants were seated approximately 26 inches $(66 \mathrm{~cm})$ from the device. Next, participants were presented with detailed PowerPoint slide instructions explaining the decision task, the alternatives and attributes, and predictive validity. The example indicated that "if an attribute has a predictive validity of 0.80 , that means that in a set of three movies, if two movies do not have the attribute, and

\footnotetext{
${ }^{4}$ These basic tables were transformed using complete row permutation resulting in six blocks consisting of a unique permutation of the five basic tables. The order and location of the row and column headings remained the same for all matrices.
} 
$\mathbf{a}$

\begin{tabular}{|c|c|c|c|c|}
\hline & Stars & Budget & Rating & Original \\
\hline Movie A & + & & & \\
\hline Movie B & + & - & - & + \\
\hline Movie C & & + & + & + \\
\hline
\end{tabular}

b

Table 1

Stars Budget Rating Original

Movie A

\begin{tabular}{|c|c|c|c|c|}
\hline \multicolumn{1}{c}{$(.8)$} & $(.7)$ & $(.6)$ & $(.5)$ & $w S u m$ \\
\hline+ & - & - & - & .8 \\
\hline- & - & + & + & 1.1 \\
\hline- & + & - & + & 1.2 \\
\hline
\end{tabular}

Table 2

\begin{tabular}{|c|c|c|c|c|}
\multicolumn{1}{c}{ Stars } & \multicolumn{1}{c}{ Budget } & \multicolumn{1}{c}{ Rating } & \multicolumn{1}{c|}{ Original } \\
\hline+ & - & + & + & 1.9 \\
\hline+ & + & - & - & 1.5 \\
\hline- & + & + & + & 1.8 \\
\hline
\end{tabular}

Table 3

\begin{tabular}{|c|c|c|c|c|}
\multicolumn{1}{c}{ Stars } & \multicolumn{1}{c}{ Budget } & \multicolumn{1}{c|}{ Rating } & Original \\
\hline+ & - & - & + & 1.3 \\
\hline- & + & + & + & 1.8 \\
\hline+ & - & - & - & .8 \\
\hline
\end{tabular}

Movie A

Movie B

Movie C

Stars Budget Rating Original

Table 4

Movie A

Movie B

Movie C

\begin{tabular}{|c|c|c|c|c|}
\multicolumn{1}{c}{ Stars } & \multicolumn{1}{c}{ Budget } & Rating & Original \\
\hline- & - & + & + & 1.1 \\
\hline- & + & - & - & .7 \\
\hline+ & - & - & - & .8 \\
\hline
\end{tabular}

Fig. 1 a. Schematic of decision task trial where options (i.e., movies) are presented in rows and attributes (e.g. Budget) are presented in columns. All cell information remains hidden (denoted in gray) until the person fixates on specific cell to reveal the cell information (denoted in black). During the task, gray information was completely occluded and only one cell was revealed at a time (i.e., budget for Movie $\mathrm{C}$ in this example). b. Matrix tables and corresponding stimuli used in experimental trials with strategy indicated per choice. We use Table 1 to illustrate between LEX and WADD strategy: Choosing Movie A represents a LEX strategy because the first attribute "Stars" with the highest attribute predictive validity distinguishes between options. Choosing Movie $\mathrm{C}$ represents a WADD strategy because the sum of the weighted attributes (illustrated as wSUM column) is highest for Movie C

two practice trials with no time restrictions. After navigating the table, the participants pressed the spacebar to advance to the choice screen where movie labels (Movie A, Movie B, and Movie C) appeared and participants pressed the 1, 2, or 3 key on the number pad (labeled $\mathrm{A}, \mathrm{B}$, and $\mathrm{C}$, respectively) to indicate their choice. The experimenter made certain the participants understood the task before starting and remained in the room during the task. screen to reveal the cell information. Participants completed 
Participants were pseudo-randomly assigned to a decreasing time ( $18 \mathrm{~s}$ to $3 \mathrm{~s}$ ) or increasing time ( $3 \mathrm{~s}$ to $18 \mathrm{~s}$ ) condition to ensure a roughly equal distribution of working memory abilities per condition. All participants also completed the baseline block with no time restrictions. For the increasing time condition, participants completed the baseline block after the last time block (after $18 \mathrm{~s}$ ), whereas in the decreasing time condition, the baseline block was completed first (before $18 \mathrm{~s}$ ).

\section{Results}

We analyzed ultimate choices (diagnostic of strategy use) and several indices of attentional information processing during the deliberation process to examine whether working memory and/or application of time (increasing or decreasing condition) accounted for differences in decision strategies across time. We used 2 (WM: HWM or LWM) $\times 2$ (Condition: increasing or decreasing time) $\times 6$ (Decision Time: $18 \mathrm{~s}, 15 \mathrm{~s}, 12 \mathrm{~s}, 9 \mathrm{~s}$, $6 \mathrm{~s}$, and $3 \mathrm{~s}$ ) mixed factorial ANOVAs for each dependent variable. We first present the behavioral data (choices) followed by information search patterns to capture decision making strategies, and then present the eye-tracking data. As a preview, differences emerged for choices, strategies and information search processing. Smaller differences were noted using eye-tracking variables indicating that using attentional processing variables to examine complex decision making might be more informative than basic differences in attention to the task.

Choice behavior and strategy To quantify choice options in line with a WADD strategy, we calculated a difference score by subtracting the number of WADD-inconsistent choices from WADD-consistent choices, and then divided by the number of trials for each level of time. Scores could range from 1 (all WADD choices) to -1 (no WADD choices). Figure 2 presents the mean WADD score for each condition and decision time by working memory group.

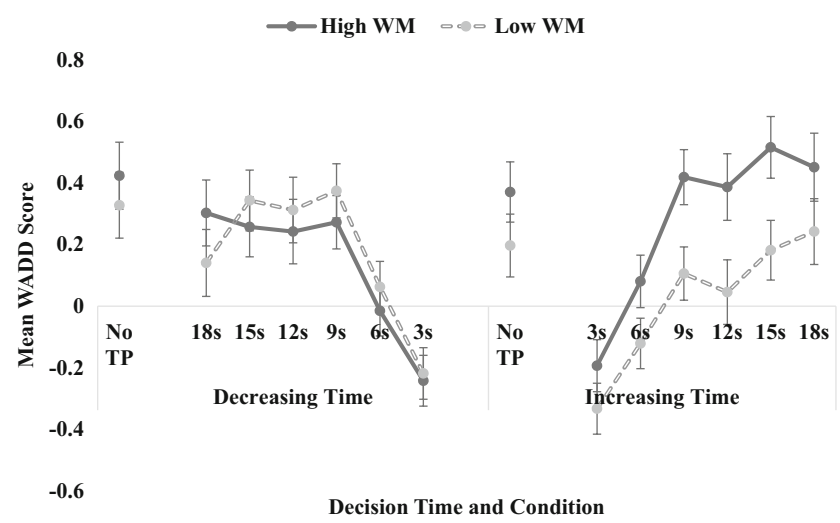

Fig. 2 Mean (standard error) WADD score ((WADD consistent - WADD inconsistent) / all choices) by decision time and condition per working memory group
The mixed factorial ANOVA revealed a significant interaction between WM and Condition, $F(1,125)=5.62, p<$ $0.05, \eta_{p}^{2}=0.04$. Inspection of Fig. 2 indicates that WM groups differed for the increasing time condition and were similar for decreasing time condition. As expected, the main effect of Decision Time was statistically significant, $F(5,125)$ $=32.41, p<0.01, \eta_{p}^{2}=0.21$, indicating that reducing the amount of time to complete the task affected choices, with less WADD use under the most restricted time constraints. The main effect of WM and condition were not statistically significant.

Additionally, we examined whether there were choice differences for the baseline block. We conducted a separate WM by order analysis for the baseline block and found no differences between working memory groups or condition, $p \mathrm{~s}>0.05$.

Strategy use consistency Because we are interested in whether or not people can learn and apply a consistent strategy even in the presence of time constraints, we classified participants based on their choices into several categories according to their ability (or not) to use a consistent strategy in most of the decision trials, namely, if they used the same strategy WADD or LEX for the majority of trials $(\geq 19 / 28)$. Table 1 presents this allocation of strategy use: WADD and LEX consistent strategies by WM group and condition. A factorial logistic regression revealed a significant interaction between WM and condition, $\chi^{2}(1)=3.68, p=0.05$. Main effects of condition and WM were not significant. Interestingly, very few participants used LEX on a consistent basis, and many were able to use a WADD strategy. As noted in the prior interaction, the apparent advantage for the HWM group is noted by the propensity for more HWM than LWM individuals to apply a consistent WADD strategy in the increasing time condition. However, our results also support that LWM can and do engage in complex strategy use.

Information search Typically, a search pattern index is used to examine how information search occurs (Payne et al., 1988). This index specifies whether transitions across table cells occur primarily within an alternative using multiple

Table 1 Percentage of participants with consistent LEX or WADD strategy use by WM and condition

\begin{tabular}{lccccc}
\hline & \multicolumn{3}{l}{ Condition and WM group } & \\
\cline { 2 - 3 } & \multicolumn{2}{l}{ Increasing time } & & \multicolumn{2}{l}{ Decreasing time } \\
\cline { 2 - 3 } \cline { 5 - 6 } & HWM & LWM & & HWM & LWM \\
\hline LEX & $3 \%$ & $12 \%$ & & $3 \%$ & $3 \%$ \\
WADD & $55 \%$ & $24 \%$ & & $33 \%$ & $41 \%$ \\
Mixed & $42 \%$ & $64 \%$ & & $64 \%$ & $56 \%$ \\
\hline
\end{tabular}


attributes (i.e., WADD strategy) or across alternatives, comparing one attribute at a time for each option (i.e., LEX strategy). The former would be denoted as search across rows represented by values from 0 to +1 and the latter along columns represented by values from -1 to 0 with larger absolute values indicative of greater consistency with the associated search mode. Inspection of Fig. 3 indicates information search occurred mainly along rows (reflected in mean values $>0$ ), which might be in part due to people typically reading from left to right; however, we did find that task variables also influenced information search. A significant WM by Condition interaction occurred, $F(1,125)=3.96, p<0.05$, $\eta_{\mathrm{p}}^{2}=0.03$, where HWM individuals tended to more consistently search across rows than LWM individuals, especially in the increasing time condition whereas no difference emerged in the decreasing time condition. This is consistent with the choices observed. Hence, the information search pattern index is reflected in the choices that an individual makes with more WADD choices chosen when searches occur across attributes within an alternative. A similar interaction by WM and condition was observed for the baseline block, $F(1,125)=5.31$, $p<0.05, \eta_{\mathrm{p}}^{2}=0.04$

Furthermore, a significant interaction emerged between decision time and condition, $F(5,121)=7.88, p<0.01, \eta_{\mathrm{p}}^{2}=$ 0.06 for search patterns. As expected, we observed greater consistency when participants were incrementally allotted more time, especially in the increasing time condition. However, in the decreasing time condition, participants search consistency increased systematically despite being exposed to increasingly greater time constraints. One would anticipate that using a WADD strategy should be correlated with positive row-wise information search (Table 2); indeed these variables are correlated. However, the application of the stressor affects the degree of the relationship strength which provides further evidence for the decision time by order interaction observed. Furthermore, the main effects of WM group, $F(1,125)=5.29$,

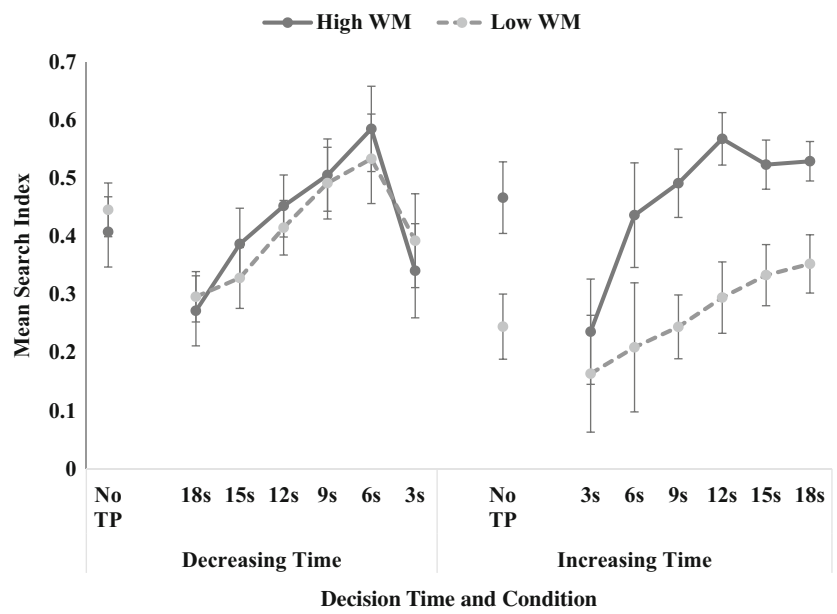

Fig. 3 Mean (standard error) search pattern index by decision time and condition per working memory group
Table 2 Correlations between WADD score with information search index and number of fixations for each level of decision time

\begin{tabular}{llllll}
\hline & \multicolumn{2}{l}{ Search index } & & \multicolumn{2}{l}{ Number of Fixations } \\
\cline { 2 - 3 } \cline { 5 - 6 } \cline { 5 - 6 } & $\begin{array}{l}\text { Decreasing } \\
\text { time }\end{array}$ & $\begin{array}{l}\text { Increasing } \\
\text { time }\end{array}$ & & $\begin{array}{l}\text { Decreasing } \\
\text { time }\end{array}$ & $\begin{array}{l}\text { Increasing } \\
\text { time }\end{array}$ \\
\hline $18 \mathrm{~s}$ & $0.46^{* *}$ & $0.38^{* *}$ & & $0.30^{*}$ & 0.22 \\
$15 \mathrm{~s}$ & $0.31^{*}$ & $0.51^{* *}$ & & 0.03 & 0.24 \\
$12 \mathrm{~s}$ & $0.42^{* *}$ & $0.37^{* *}$ & & 0.09 & 0.11 \\
$9 \mathrm{~s}$ & $0.30^{*}$ & $0.40^{* *}$ & & 0.10 & 0.01 \\
$6 \mathrm{~s}$ & 0.19 & $0.30^{*}$ & & $0.29^{*}$ & 0.24 \\
$3 \mathrm{~s}$ & $0.25^{*}$ & $0.51^{* *}$ & & $0.28^{*}$ & 0.05 \\
$\mathrm{No} \mathrm{TP}$ & $0.29^{*}$ & $0.30^{*}$ & & -0.07 & -0.08 \\
\hline
\end{tabular}

$* p<0.05 ; * *<0.01$

$p<0.05, \eta_{\mathrm{p}}^{2}=0.04$, and decision time, $F(5,121)=5.07$, $p<0.01, \eta_{\mathrm{p}}^{2}=0.04$, also were statistically significant.

In sum, the information search results indicated greater consistency and searching across attributes within an alternative, which is consistent with a WADD strategy. Both WM groups were able to maintain more consistent search when they started with more time to choose (decreasing time condition); however, differences in WM emerged with an advantage for HWM individuals when they started with a restrictive time constraint and were allowed more time as they progressed through the experiment. The LWM individuals did not appear to adapt to the same degree as the HWM individuals for the increasing time condition. As such, choice and search indices reveal differences in WM group as a function of how the time pressure is applied.

Fixations and duration A fixation was computed from the onset of eye movement to an area of interest (AOI) until the eye movement was displaced from the given AOI, using the raw eye-tracking data. The fixation duration reflected the time (ms) spent on each AOI. Figure 4a presents average number of fixations, and Fig. 4b presents average fixation duration. Analysis of fixations revealed that the main effect of WM group, $F(1,125)=3.85, p=0.05, \eta_{\mathrm{p}}^{2}=0.03$, with HWM individuals demonstrating the tendency to have more fixations than the LWM individuals. Figure 4a shows participants had fewer fixations for extreme time constraints (as expected) compared with liberal time constraints, which resulted in a significant main effect of Decision Time, $F(5,121)=$ 917.78, $p<0.01, \eta_{\mathrm{p}}^{2}=0.88$. The main effect of condition and the interactions were not statistically significant. Thus, the time pressure had the intended effect to reduce the ability to attend to information within the table, irrespective of order. The trend appeared to provide a slight advantage for HWM individuals in terms of fixations, albeit not statistically significant. A separate analysis for the baseline block found no 


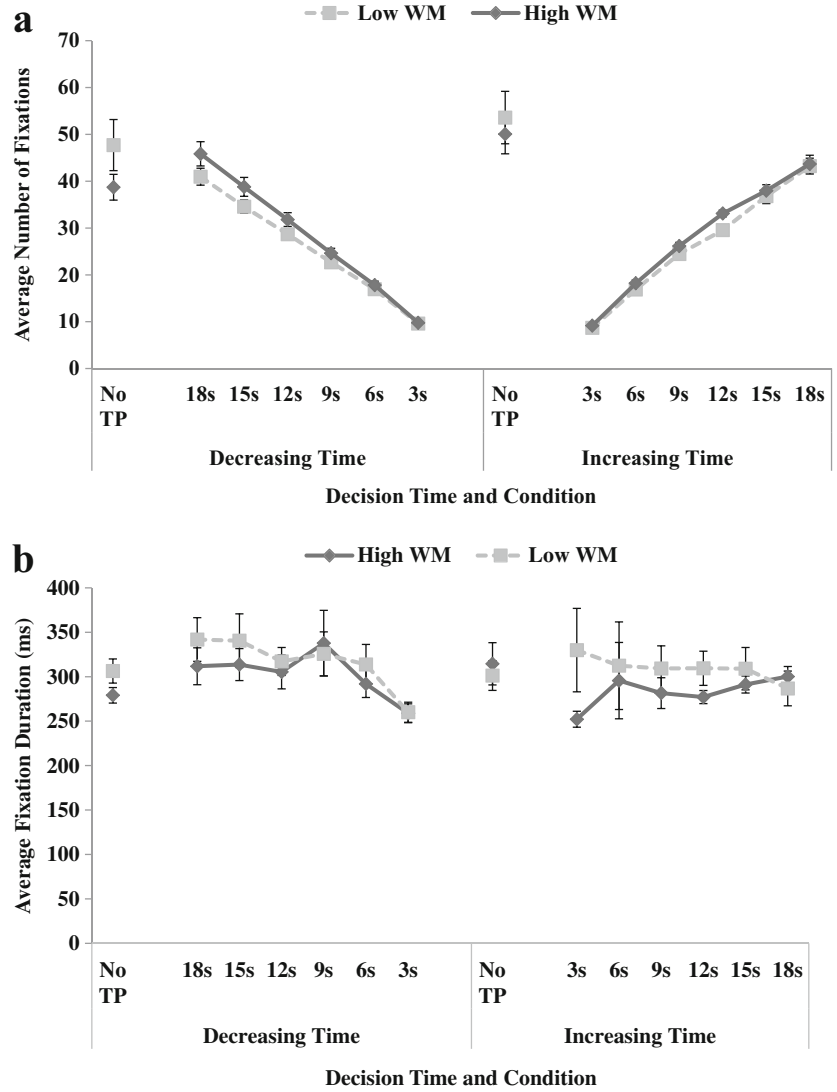

Fig. 4 a. Mean (standard error) number of fixations by decision time and condition per working memory group. b. Mean (standard error) fixation duration $(\mathrm{ms})$ by decision time and condition per working memory group

differences due to working memory group or time pressure order, $p>0.05$. Although one might anticipate more fixations associated with WADD strategy use, these correlations were modest when present (Table 2), and no consistent pattern between WADD use and average number of fixations emerged.

For fixation duration, a significant time $\times$ condition interaction was present, $F(5,121)=2.43, p<0.05, \eta_{\mathrm{p}}^{2}=0.02$. The decreasing time condition had slightly longer durations in the liberal time constraints and dropped off at the strictest time constraints, whereas the increasing time condition resulted in similar durations regardless of the amount of time allotted. The main effect of time was statistically significant, $F(5$, $121)=3.02, p<0.05, \eta_{p}^{2}=0.02$. No other main effects or interactions were significant. The baseline block did not yield a significant difference between working memory groups or order, $p \mathrm{~s}>0.05$. No correlations were observed between WADD strategy use and average fixation duration.

In sum, the attentional processing variables fixations and duration revealed minimal or no differences between WM groups despite the fact that choice behavior and search indices mattered in the increasing time condition.

Attention variables and choice Lastly, we examined whether attentional processing variables predicted choice behavior. For each level of time, we computed a step-wise regression by first inputting fixations, durations, and cells accessed (i.e., proportion of cells within the 12-cell matrix examined) and then entered search pattern index to determine whether the basic attention (step 1) and attention search (step 2) accounted for greater WADD strategy as measured with our WADD score.

Table 3 reveals the results of the regressions. As noted in the table, overall the attention variables contributed to significantly predict choice, albeit, the best predictor was cells accessed, which is consistent with the increased information

Table 3 Step-wise regressions: model and $\beta$ values for attention variables (fixations, durations, and cells accessed) (step 1) and search pattern index (step 2) to predict WADD score per level of time

\begin{tabular}{|c|c|c|c|c|c|c|}
\hline Models and variables & Time & & & & & \\
\hline Step 1 & $18 \mathrm{~s}$ & $15 \mathrm{~s}$ & $12 \mathrm{~s}$ & $9 \mathrm{~s}$ & $6 \mathrm{~s}$ & $3 \mathrm{~s}$ \\
\hline Cells accessed & $0.430 * *$ & $0.551 * *$ & $0.483 * *$ & $0.469 * *$ & $0.367 * *$ & 0.195 \\
\hline Fixations & 0.050 & -0.033 & -2.00 & -0.072 & $0.281 *$ & 0.121 \\
\hline Durations & 0.003 & 0.182 & -0.055 & 0.264 & $0.418 * *$ & 0.168 \\
\hline$F$ & $10.809 * *$ & $8.679 * *$ & $2.566^{* *}$ & $3.340 *$ & $7.837 * *$ & 2.367 \\
\hline$R^{2}$ & 0.206 & 0.172 & 0.162 & 0.074 & 0.158 & 0.054 \\
\hline \multicolumn{7}{|l|}{ Step 2} \\
\hline Cells accessed & $0.288^{*}$ & $0.377 * *$ & 0.256 & 0.280 & 0.278 & -0.006 \\
\hline Fixations & 0.090 & -0.014 & -0.238 & -0.097 & $0.268 *$ & 0.153 \\
\hline Durations & -0.012 & 0.153 & -0.133 & 0.198 & 0.294 & 0.017 \\
\hline Search index & $0.309 * *$ & $0.266^{* *}$ & $0.273^{* *}$ & $0.325 * *$ & 0.154 & $0.379 * *$ \\
\hline$F$ & $12.484 * *$ & $8.870^{* *}$ & $8.259 * *$ & $5.733 * *$ & $6.572 * *$ & $6.433^{* *}$ \\
\hline$R^{2}$ & 0.287 & 0.222 & 0.210 & 0.156 & 0.175 & 0.172 \\
\hline$\Delta R^{2}$ & $0.081 * *$ & $0.050^{* *}$ & $0.049 * *$ & $0.082 * *$ & 0.017 & $0.118^{* *}$ \\
\hline
\end{tabular}

Standardized $\beta$ values indicated: $* * p<0.01, * p<0.05, p<0.10$ 
required for utilizing a WADD strategy. Step 2 models revealed that search index, which is information processing in a row or column fashion within the table matrix, predicted choice above and beyond cells accessed. Thus, we see evidence of both attention and information processing impacting strategy use.

\section{Discussion}

As anticipated, time constraints impacted how individuals acquire information and utilize strategies, with a tendency for less complex strategy use during the most restrictive conditions (Rieskamp \& Hoffrage, 2008). However, some individuals were able to maintain consistent WADD strategy use even during more severe time restrictions, which aligns with prior work (Glöckner \& Betsch, 2008). We found convergence between information search and strategy use, especially for individuals who maintained consistent WADD strategy use despite the levels of time pressure. The application of decision time affected strategy use whereby a shift from a simple to a more complex strategy was observed when more time was allowed. Differences in both choice behavior and information search emerged between WM groups in the increasing time condition. As such, lower WM individuals appeared to persist with a LEX strategy longer and engage in a column-wise search pattern more often than those with higher WM. This is consistent with work where individual differences in WM emerge when pressure is lessened or no stressor is present (Beilock \& Carr, 2005; Engle, 2002). However, past research has not systematically examined how the application of the stressor (increasing or decreasing time in an incremental manner) affects choice, information search, and strategy use.

Interestingly, with regards to strategy use and information search, decreasing time had the same effect for all participants, irrespective of working memory. In this condition, we observed that when allowed enough time, most individuals, including those with lower WM will use a WADD strategy. These results indicate that perhaps gradually introducing individuals to more severe stressors will enable them to persist in using a complex strategy in spite of the stressor demands on attentional processing.

We found that the attentional processing variables (fixations and duration) revealed minimal or no differences between WM groups. Thus, it would appear that attentional processing was not the driving factor between differences in WM to strategy use and choice behavior. As such, the possibility that individuals with lower WM are less able than high WM individuals to acquire the information is ruled out. We did observe differences in search patterns and choices when decision time increased and low WM individuals did not transition to using complex strategies to the same degree as the high WM individuals.
We might attribute this absence of large differences in attentional and information processing between working memory groups to the use of binary cues in our task. Specifically, simpler binary cues might not have placed enough demands on working memory above and beyond the time demands associated with the task. The simpler cues may have contributed to neutralizing the typical advantage for higher WM individuals. By imposing more cognitive demands in the decision task, such as using more complex cues or by varying categorical information, differences in working memory are more likely to emerge if they exist at the attentional processing level.

This research built upon and extended prior work demonstrating how deliberate information processing, attention, and strategy use can be better understood through the lenses of individual differences and gradations in applications of time pressure.

\section{Conclusions}

Perhaps in situations that require making decisions under time restrictions, one can influence performance by being sensitive to working memory and, potentially, by allowing an acclimation period to adapt to stringent task time through progressive decreases in time. An acclimation period may serve to allow some individuals to engage in complex strategies even under severe time constraints, where these individuals would not otherwise be able to engage in the strategies had an acclimation period not been provided.

\section{References}

Ashby, N. J. S., \& Rakow, T. (2014). Forgetting the past: Individual differences in recency in subjective valuations from experience. Journal of Experimental Psychology: Learning, Memory, and Cognition, 40, 1153-1162.

Awh, E., Vogel, E. K., \& OH, S.-H. (2006). Interactions between attention and working memory. Neuroscience, 139, 201-208.

Baddeley, A. D. (2002). Is working memory still working? European Psychologist, 7, 85-97.

Baddeley, A., \& Hitch. (1974). Working memory. In G. A. Bower (Ed.), Recent advances in learning and motivation (Vol. 8, pp. 47-90). New York: Academic Press.

Beach, L. R., \& Mitchell, T. R. (1978). A contingency model for selection of decision strategies. Academy of Management Review, 3, 439-449.

Beilock, S. L., \& Carr, T. H. (2005). When high-powered people fail. Working memory and "chocking under pressure" in math. Psychological Science, 16, 101-105.

Beilock, S. L., \& DeCaro, M. S. (2007). From poor performance to success under stress: Working memory, strategy selection, and mathematical problem solving under pressure. Journal of Experimental Psychology: Learning, Memory, and Cognition, 33, 983-998.

Ben-Zur, H., \& Breznitz, S. J. (1981). The effect of time pressure on risky choice behavior. Acta Psychologica, 47, 89-104. 
Böckenholt, U., \& Kroeger, K. (1993). Time pressure effects in multiattribute binary choices. In J. Maule \& O. Svenson (Eds.), The effects of time pressure and stress in human judgment and decision making (pp. 195-214). New York: Plenum Press.

Dougherty, M. R. P., \& Hunter, J. E. (2003). Hypothesis generation, probability judgment, and individual differences in working memory capacity. Acta Psychologica, 113, 263-282.

Dougherty, M. R., \& Sprenger, A. (2006). The influence of improper sets of information on judgment: How irrelevant information can bias judged probability. Journal of Experimental Psychology: General, $135,262-281$.

Engle, R. W. (2002). Working memory capacity as executive attention. Current Directions in Psychological Science, 11, 19-23.

Fiedler, S., \& Glöckner, A. (2012). The dynamics of decision making in risky choice: An eye-tracking analysis. Frontiers in Psychology, 3, $1-18$.

Fink, A., \& Neubauer, A. C. (2005). Individual differences in time estimation related to cognitive ability, speed of information processing and working memory. Intelligence, 33(1), 5-26.

Franco-Watkins, A. M., \& Johnson, J. G. (2011a). Decision moving window: Using eye tracking to examine decision processes. Behavior Research Methods, 43, 853-863.

Franco-Watkins, A. M., \& Johnson, J. G. (2011b). Applying the decision moving window to risky choice: Comparison of eye-tracking and mouse-tracing methods. Judgment and Decision Making, 6, 740 748.

Franco-Watkins, A. M., Mattson, R. E., \& Jackson, M. J. (2016). Now or later? Attentional processing in intertemporal choice. Journal of Behavioral Decision Making, 29, 206-217. doi:10.1002/bdm.1895

Glöckner, A., \& Betsch, T. (2008). Multiple-reason decision making based on automatic processing. Journal of Experimental Psychology: Learning, Memory, and Cognition, 34, 1055-1075.

Kane, M. J., Bleckley, M. K., Conway, A. R. A., \& Engle, R. W. (2001). A controlled-attention view of working-memory capacity. Journal of Experimental Psychology: General, 130, 169-183.

Kane, M. J., \& Engle, R. W. (2000). Working memory capacity, proactive interference, and divided attention: Limits on long-term memory retrieval. Journal of Experimental Psychology: Learning, Memory, \& Cognition, 26, 333-358.

Kerstholt, J. H. (1995). Decision making in a dynamic situation: The effect of false alarms and time pressure. Journal of Behavioral Decision Making, 8, 181-200.

Lewandowsky, S. (2011). Working memory capacity and categorization: Individual differences and modeling. Journal of Experimental Psychology-Learning Memory and Cognition, 37, 720-738.
Lohse, G. E., \& Johnson, E. J. (1996). A comparison of two process tracing methods for choice tasks. Organizational Behavior and Human Decision Processes, 68, 28-43.

Maule, A. J. (1994). A componential investigation of the relation between structural modelling and cognitive accounts of human judgement. Acta Psychologica, 87, 199-216.

Maule, A. J., Hockey, G. R. J., \& Bdzola, L. (2000). Effects of time pressure on decision making under uncertainty: Changes in affective state and information processing strategy. Acta Psychologica, 104, 283-301.

Payne, J. W., Bettman, J. R., \& Johnson, E. J. (1988). Adaptive strategy selection in decision making. Journal of Experimental Psychology: Learning, Memory, and Cognition, 14, 534-552.

Rakow, T., Newell, B. R., \& Zougkou, K. (2010). The role of working memory in information acquisition and decision making: Lessons from the binary prediction task. Quarterly Journal of Experimental Psychology, 63, 1335-1360.

Rieskamp, J., \& Hoffrage, U. (2008). Inferences under time pressure: How opportunity costs affect strategy selection. Acta Psychologica, 127, 258-276.

Russo, J. E., \& Leclerc, F. (1994). An eye-fixation analysis of choice processes for consumer nondurables. Journal of Consumer Research, 21, 274-290.

Russo, J. E., \& Rosen, L. D. (1975). An eye fixation analysis of multialternative choice. Memory \& Cognition, 3, 267-276.

Sanchez, C. A., \& Wiley, J. (2006). An examination of the seductive details effect in terms of working memory capacity. Memory \& Cognition, 34, 344-355.

Sprenger, A. M., Dougherty, M., Atkins, S. M., Franco-Watkins, A. M., Thomas, R., Lange, N., \& Abbs, B. (2011). Implications of cognitive load for hypothesis generation and probability judgment. Frontiers of Cognitive Science, 2, 1-15.

Svenson, O., \& Maule, A. J. (Eds.). (1993). Time pressure and stress in human judgment and decision making. New York: Plenum Press.

Unsworth, N., Brewer, G. A., \& Spillers, G. J. (2013). Working memory capacity and retrieval from long-term memory: The role of controlled search. Memory \& Cognition, 41, 242-254.

Unsworth, N., Heitz, R. P., Schrock, J. C., \& Engle, R. W. (2005). An automated version of the operation span task. Behavior Research Methods, 37, 498-505.

Weenig, M. W., \& Maarleveld, M. (2002). The impact of time constraint on information search strategies in complex choice tasks. Journal of Economic Psychology, 23(6), 689-702.

Wright, P. (1974). The harassed decision maker: Time pressures, distractions, and the use of evidence. Journal of Applied Psychology, 59, $555-561$. 